\title{
RADIATIVE TRANSFER PROCESSES IN PLANETARY \\ NEBULAE
}

\author{
D. G. HUMmer \\ ( Joint Institute for Laboratory Astrophysics, U.S.A.)
}

\begin{abstract}
1.0. Introduction
The physical aspects of radiative transfer processes that may be of importance in planetary nebulae are discussed, and recent work on these problems is summarized.
\end{abstract}

\subsection{The Continuum Photo-ionization-Recombination Problems}

The fundamental radiative transfer problem in planetary nebulae is that of the hydrogen Lyman continuum, which also serves as the prototype for continuum transfer problems involving other elements. In the simplest form of this problem, in which only hydrogen is present, extremely dilute Lyman-continuum radiation from the central star falls on the inner boundary of the nebula, photo-ionizing the hydrogen. A certain fraction of the ionizations are followed by recombinations to the ground state, with the production of 'diffuse' Lyman-continuum photons. Because the diffuse fleld is roughly isotropic and has sources within the nebula, while the stellar field is radial and satisfies a simple transfer equation, it is convenient to maintain this division of the total continuum radiation field. There are two cardinal facts relating to the ionization-recombination process in planetary nebulae:

(1) The probability that a Lyman-continuum photon will be emitted in a recombination is approximately $0 \cdot 4$.

(2) The spectral distribution of the diffuse Lyman-continuum photons is concentrated very strongly to the immediate short-wave side of the Lyman limit, while the original distribution is much wider.

The importance of the first point, which was recognized by Ambartsumian (1932), is that the absorption of a typical Lyman-continuum photon will be followed by only a few photo-ionization-recombination cycles before all of the photon energy is degraded into line radiation. If we visualize this process as the repeated absorption and emission of a photon, we can say that a diffuse photon can move only a few free paths from its point of creation. This diffuse photon will, of course, change its frequency at each scattering because of the 'reshuffling' of the continuum states, a process first treated in this context by Baker et al. (1939). The second point indicates 
that diffuse photons see the maximum photo-ionization cross-section, i.e. their free paths are as short as possible.

Zanstra (1951a), recognizing that these points together imply that a diffuse photon does not travel very far before being converted into line photons, introduced a very useful approximation: that the diffuse photons are degraded at the point where they are created. This so-called on-the-spot (OS) approximation reduces the transfer problem to the evaluation of simple integrals, which have been tabulated by De Jong (1951) and Hummer and Seaton (1963) for hydrogen, and by Hummer and Seaton (1964) for ionized helium. In particular, the OS approximation is much easier to apply than is the method used by Aller et al. (1939), in which an integro-differential equation for the diffuse radiation field is solved approximately by iteration. The OS approximation can also be applied to the ionization-recombination problem for other elements. Necessary conditions for its validity are

(1) The probability per recombination that a continuum photon, as opposed to line photons, is produced must be small, and

(2) The electron temperature must be low enough for $k T_{\mathrm{e}}$ to be small in comparison to the ionization energy.

A further condition for the validity of the OS approximation may be obtained by estimating the Lyman-limit optical distance between the point where a typical diffuse photon is created and the point where it finally is degraded into line photons. We refer to this distance as the 'break-up length' and represent it by $\Lambda$. Obviously photons created within an optical distance $\Lambda$ of a boundary will most likely escape before being degraded, so the OS approximation fails there. If the optical thickness of the nebula is of the same order as $\Lambda$, the OS approximation fails everywhere. Thus a third condition for the validity of the OS approximation is that the optical thickness be larger than $\Lambda$.

Van Blerkom and Hummer (1967), in analogy with an unpublished expression derived by Rybicki for line scattering, have obtained the result

where

$$
\Lambda=l / \sqrt{\varepsilon}
$$

$$
l^{2}=2 \int_{v_{1}}^{\infty}\left[f\left(v / v_{1}\right)\right]^{-1} B_{v}\left(T_{\mathrm{e}}\right) \mathrm{d} v / \int_{v_{1}}^{\infty} f\left(v / v_{1}\right) B_{v}\left(T_{\mathrm{e}}\right) \mathrm{d} v
$$

and

$$
\varepsilon=1-\left(\alpha_{1} / \alpha_{\mathrm{tot}}\right)
$$

Here $f(v)$ is the photo-ionization cross-section normalized to unity at the Lyman limit $v_{1}$; and $\alpha_{1}$ and $\alpha_{\text {tot }}$ are, respectively, the recombination coefficients to the ground state and to all states. Using Kramers' approximation, $f=\left(v / v_{1}\right)^{-3}$, we have

$$
l^{2} \simeq{ }_{3}^{2}\left\{1+6\left(\begin{array}{c}
k T_{\mathrm{e}} \\
h v_{1}
\end{array}\right)+24\left(\begin{array}{c}
k T_{\mathrm{e}} \\
h v_{1}
\end{array}\right)^{2}+\cdots\right\}, \quad h v_{1} \gg k T_{\mathrm{e}} .
$$


If the nebula is sufficiently thin, the diffuse radiation is much weaker than the direct stellar radiation and the ionization balance is reasonably accurate, even if the OS approximation is not. Because of the evidence given by Harman and Seaton (1966), that planetary nebulae are not completely opaque in the Lyman continuum for a substantial fraction of their lives, Van Blerkom and Hummer (1967) have derived a modified OS approximation which makes some allowance for the boundaries and can be used for all optical thicknesses. Comparison with accurate numerical solutions indicates that, for the conditions of planetary nebulae, the error in the radiation field in the improved approximation is less than $10 \%$. This approximation turns out to coincide with that obtained by bringing the source function through the integral sign in the integral form of the transfer equation, an approximation used earlier by Biberman (1948) and by Sobolev (1957) for line transfer problems (a rather unreliable procedure) and by Biberman et al. (1965) for continuum problems. This work has also confirmed that diffuse radiation entering the nebula from the opposite hemisphere is generally unimportant.

For nebulae sufficiently thick so that all Lyman-continuum radiation is absorbed, the OS approximation is considerably more accurate than the well-known formula developed by Strömgren (1939). Although the radius of the ionized region agrees with Strömgren's estimate, the very sharp transition region is smoothed out because the stellar photons with high energies see a comparatively small photo-ionization crosssection and also because of the diffuse radiation. A further point, which has recently emerged, is that the size of the ionized region and the degree of ionization depend primarily on the total number of ionizing photons entering the nebula and only weakly on the spectral distribution.

The effects of electron collisions on the ionization and thermal equilibrium and on the distribution of excited states have been considered by a number of authors, the most recent being Chamberlain (1953), Hummer (1963a), and Parker (1964). When the nebula is excited by a central star, then collisional excitations and ionization are unimportant compared to photo-ionization and recombination, except perhaps in the thermal balance of the outermost regions. In situations where the primary excitation mechanism is collisional, collisions from the ground state determine the state of excitation and ionization in optically thin nebulae. However, Van Blerkom (1968) has shown that diffuse radiation becomes important in populating excited states if the nebula is thick.

\subsection{Models including Helium and Heavy Elements}

The picture becomes considerably more complicated when helium and heavier elements are added to the hydrogen. In the first place, the ionization continua of the different elements overlap, so that in many spectral regions two or more elements are competing for the same radiation. The second problem is that radiation emitted in the 
recombination and cascade of highly ionized systems can ionize species in lower stages of ionization, the most important example of which is the ionization of hydrogen and helium by He II Ly- $\alpha$. From a technical point of view, both of these effects lead to severely non-linear transfer problems. Additional complications arise from fluorescent mechanisms, such as that discovered by Bowen, caused by the coincidence of lines of different elements.

The first solution for a combined hydrogen-helium model was obtained by Eberlein $(1955,1957)$, who used a differential formulation of the Strömgren theory with a mean absorption coefficient to determine the ionization balance between $\mathrm{He}^{+}$and $\mathrm{He}^{++}$, along with that for hydrogen. He obtained the radii of the ionization zones and the $\lambda 4686 \AA / \mathrm{H} \beta$ ratio as a function of the relative abundance of helium. Osaki (1962) also discussed a multi-component model, but as he was interested primarily in the thermal balance rather than the ionization balance, he used rather crude estimates for the ion densities.

Hummer and Seaton (1964) gave an extensive, though approximate, discussion of the hydrogen-helium problem. The $\mathrm{He}^{+}-\mathrm{He}^{++}$balance was solved using the OS approximation. Because of the fortunate circumstance that the Ly- $\alpha$ line, the two-quantum continuum, and the Balmer continuum of HeII were sufficiently strong to keep the hydrogen in the $\mathrm{He}^{++}$-zone fully ionized, the stellar radiation with $v<4 v_{1}$ could, to a first approximation, be regarded as passing through this zone unimpeded. Although the spectral distribution would be altered somewhat, the number of photons available for the ionization of $\mathrm{H}$ and $\mathrm{He}$ beyond the $\mathrm{He}^{++}$zone would be correct. Beyond the $\mathrm{He}^{++}$zone, the ionization equilibria involving neutral hydrogen and neutral helium in this radiation field were solved using a differential formulation of the OS approximation (capable of handling any number of elements and stages of ionization). Probably the most important result of this investigation was that for star temperatures below about $50000^{\circ} \mathrm{K}$ the helium spectrum reflects the stellar temperature, while at higher temperatures the helium spectrum depends only on the helium abundance.

The most complete treatment published to date is that of Goodson (1967), who solved the combined ionization and thermal balance problems, including $\mathrm{H}, \mathrm{He}, \mathrm{C}$, $\mathrm{N}, \mathrm{O}$ and $\mathrm{Ne}$, using the stellar fluxes computed by Böhm and Deinzer $(1965,1966)$. Goodson used the OS approximation as an initial estimate for an iterative procedure, although the results of the iterative calculation did not differ greatly from the OS results. Probably the least satisfactory aspect of Goodson's work is his treatment of HeII Ly- $\alpha$, which he assumes to be absorbed on the spot by hydrogen alone. This assumption considerably overestimates the rate of ionization of hydrogen in the $\mathrm{He}^{++}$ region and underestimates it elsewhere. It is possible that $\mathrm{HeII} \mathrm{Ly}-\alpha$ diffuses out of the region where it is created and is absorbed mainly in ionizing helium and hydrogen at the places where they are becoming neutral, thereby altering the conditions in the transition zone. Goodson's neglect of the ionization of helium by HeII Ly- $\alpha$ is probably also unjustified, since for $\mathrm{He}$ II Ly- $\alpha$ the helium photo-ionization cross-section is 
about five times that of hydrogen, compensating for the lower abundance of helium. For both of these reasons, Goodson overestimates the energy of the free electrons in the $\mathrm{He}^{++}$region at the expense of the electrons elsewhere. Although Goodson's temperatures are probably somewhat in error, the ionization equilibria are probably quite good, in the sense that they represent the stellar fluxes assumed.

More recent calculations of ionization equilibria are reported in these Proceedings by Flower and by Williams. Ionization equilibria including heavy atoms have been computed for dynamical models of Hil regions by Hjellming (1966), who employed the OS approximation.

\subsection{Lyman- $\alpha$ Lines}

The most important of the resonance-line transfer problems are those of $\mathrm{HI}, \mathrm{He}$ and HeII Ly- $\alpha$. It is convenient to regard the hydrogen line as the prototype, if only because it has received the most attention. These lines are distinguished by their large optical thickness and by the very strong sources feeding energy into them. This combination of factors leads to the possibility of very large intensities. For example, the line centre optical thickness of a planetary nebula in $\mathrm{HI}_{\mathrm{I}} \mathrm{Ly}-\alpha$ is about $10^{4}$ times that at the Lyman limit (assuming $T_{\mathrm{e}}=10^{4}{ }^{\circ} \mathrm{K}$ ) and, in the simplest picture, the line receives about $\frac{2}{3}$ of the photons in the stellar Lyman continuum.

Generally, the intensity of radiation in a line is determined by the relative rates at which photons enter and leave the line. While the rate at which photons are fed into these lines is given simply by recombination theory, the rate at which they leave depends on many factors. The most obvious method is to escape through the boundary of the nebula. However, if the optical thickness of the nebula in the line is very large, the number of scatterings necessary to escape can be extremely large, so that processes with a small probability per scattering of destroying photons can become important.

\subsubsection{ESCAPE MECHANISMS}

The rate at which photons escape from the nebula is determined mainly by four factors: noncoherent scattering, velocity gradients, thermal gradients, and blanketing by interstellar hydrogen.

\subsubsection{NONCOHERENT SCATTERING}

Henyey (1941) first pointed out that when a photon is scattered by a moving atom, its initial and final frequencies as seen by a stationary observer will in general differ because the change in the photon's direction will cause the Doppler shift to vary. This so-called noncoherence was recognized by Sobolev $(1944,1947)$ (in a paper written in 1941) and independently by Zanstra $(1949,1951 a)$ as being of primary importance 
for the Ly- $\alpha$ problem, since it allows photons created in the very opaque line core to be scattered into the relatively transparent wings where they can more readily escape. A general discussion of noncoherent scattering is given by Hummer (1962).

Two forms of noncoherent scattering are encountered in planetary nebulae. At a kinetic temperature of $10^{4} \mathrm{~K}$ (assumed equal to the electron temperature), the ratio of the natural width to the Doppler width is $3.7 \times 10^{-4}$ for $\mathrm{HI}$ Ly- $\alpha$ and $3.8 \times 10^{-3}$ for HeII Ly- $\alpha$. Within the core of the line, out to some three Doppler half-widths, the absorption coefficient is dominated by Doppler broadening, and the emission coefficient is approximately proportional to the absorption coefficient, i.e. there is no correlation between the absorption and emission frequencies. In the line wings, which are dominated by natural broadening, the scattering has a strong coherent component, because photons lying far from the line centre are scattered mainly by atoms in the low-velocity part of the Maxwellian distribution. These two cases are referred to as complete and partial redistribution, respectively. If the line-centre optical thickness is not too large, less than e.g. about $10^{4}$ for $\mathrm{HI}$ Ly- $\alpha$, then the line becomes optically thin at frequencies which are still within the Doppler core and complete redistribution can safely be assumed for the entire line.

Complete solutions of the radiative transfer problems can be obtained only by numerical methods; a very comprehensive review of these techniques is given by Hummer and Rybicki (1967). The first accurate numerical solution for complete redistribution was obtained by Koelbloed (1956) for isothermal plane-parallel nebulae with line-centre optical thickness as large as $2 \times 10^{4}$. When partial redistribution must be taken into account, the computational problem is much more difficult. The earliest attempts were too crude to yield anything but a qualitative picture of the radiation field. Unno (1951, 1955) obtained the first solutions for HI and He II Ly- $\alpha$ which were essentially correct, although not very accurate, because of the number of approximations he was forced to make. His results demonstrated clearly that while the mean intensity in the line core was quite large the flux was large only in the far wings. Hearn (1964) and Hummer (1968) have obtained accurate numerical solutions using a Cebyšev expansion technique and a generalized discrete-ordinate method, respectively, although these solutions were not obtained in the context of the nebular line problem. Auer (1968) has employed a Monte-Carlo technique specifically to study the Ly- $\alpha$ line in planetary nebulae.

For many problems it is sufficient to know the average number of scatterings experienced by a photon before leaving the nebula, or alternatively, the mean probability of escape. When complete redistribution obtains, a simple argument given by Zanstra (1949) may be used. The basic assumption is that a photon will escape if its monochromatic optical distance from the nearest surface is less than unity and that it will be re-scattered otherwise. If $\phi(v)$ is the normalized emission coefficient, equal by assumption to the normalized absorption coefficient, the probability of escape 
from a point with line-centre optical depth $\tau_{0}$ is

$$
p\left(\tau_{0}\right)=2 \int_{v_{c}}^{\infty} \phi(v) \mathrm{d} v,
$$

where $v_{c}$ is defined by

$$
\frac{\phi\left(v_{c}\right)}{\phi\left(v_{0}\right)} \tau_{0}=1 .
$$

For Doppler scattering, $\phi(v)=\exp \left(-\left(v-v_{0} / \Delta\right)^{2}\right) / \Delta \sqrt{ } \pi$ and

$$
p\left(\tau_{0}\right)=\int_{\sqrt{ } \pi}^{2} \int_{\sqrt{\ln \tau_{0}}}^{\infty} e^{-x^{2}} \mathrm{~d} x, \quad \tau_{0} \gg 1 .
$$

Strictly speaking, this argument applies only to a one-dimensional nebula, since the relevant quantity is the monochromatic optical depth measured along the photon's direction of flight and not, as assumed above, the optical depth normal to the surface. Obviously, to obtain the mean escape probability, $p\left(\tau_{0}\right)$ should be multiplied by the depth distribution of emission and the quantity averaged over depth. The objections are partially overcome by Hummer (1964), and very recently Sobolev (1967) has obtained rigorous bounds and asymptotic formulae for the mean number of scatterings. Capriotti (1965) has applied Zanstra's argument to a uniform spherical nebula expanding with a constant velocity gradient and has given a number of approximate expressions for mean escape probabilities.

When partial redistribution is important, calculation of escape probabilities or the mean number of scatterings is much harder. Osterbrock (1962), in a very important paper, has taken advantage of the fact that a typical Ly- $\alpha$ photon does not move very far from its point of creation to the point where it experiences its last scattering before escaping, i.e. the photon remains at roughly the same depth while undergoing enough frequency changes to get it into the wing from which it escapes. Osterbrock also assumes that no photons escape in the line core, which he takes as extending 3.25 Doppler half-widths on either side of the line centre. Photons arriving in the wing escape with a certain probability, otherwise scattering back into the core. By following the history of each photon on the basis of an approximate frequency-diffusion theory, Osterbrock is able to calculate the mean number of scatterings experienced by a photon created at a depth $\tau_{0}$. His results differ at most by a factor of 3 from Zanstra's conclusion that $\langle N\rangle \sim \tau_{0} \sqrt{ } \pi \ln \tau_{0}$.

It would appear that Osterbrock's estimates are probably larger than the exact values. In the first place, Auer (1968) finds that approximately $\frac{1}{4}$ of the escaping photons have frequencies in the core. If the number of scatterings is large, a core photon can diffuse a considerable distance, especially at frequencies near the somewhat arti- 
ficial division between core and wing. The outward decrease of the kinetic temperature in real nebulae also provides a bias towards preferential outward scattering. Some error also arises from Osterbrock's assumption that all photons are created at depth $\tau_{0}$, which he takes to be the optical thickness of the nebular shell. If the nebula is optically thick in the Lyman continuum, then most of the Ly- $\alpha$ photons are produced near the inner boundary and identifying $\tau_{0}$ with the optical thickness is correct. However, if the Lyman continuum is optically thin, then Ly- $\alpha$ photons are created more or less uniformly throughout the shell, so that Osterbrock's (and Zanstra's) estimates should be averaged over the shell.

The time development of the Ly- $\alpha$ line in a very thick shell has been studied in a very general manner by Field (1959) and by Ivanov (1967), on the basis of partial and complete redistribution, respectively. Ivanov has shown that the leading term in the asymptotic solutions for long times is identical for both kinds of redistribution.

\subsubsection{VELOCITY GRADIENTS}

If a nebula is expanding with a velocity gradient, the line opacity will be shifted in frequency by increasing amounts as one proceeds outwards through the nebula. After Ambartsumian's (1932, 1933) and Chandrasekhar's (1935) pioneering investigations of the Ly- $\alpha$ radiation field in static and uniformly expanding nebulae led to catastrophic radiation pressure arising from the unrealistic assumption of coherent scattering, Zanstra $(1934,1936)$ introduced the effects of velocity gradients. He also assumed coherent scattering and found a drastic reduction in the radiation pressure. Sobolev (1947) treated this problem in a more general and practical way and found that Zanstra's treatment was in error.

Sobolev's original investigation included the effects of both velocity gradients and noncoherent scattering, but was based on the rather unsatisfactory assumption of a rectangular absorption profile. However, in a later paper, which does not seem to have attracted the attention it deserves, Sobolev (1957) generalized his treatment to include an arbitrary absorption coefficient, with complete redistribution, and allowing for an arbitrary distribution of expansion velocities with depth. Most of Sobolev's results are presented for a constant velocity gradient.

Unfortunately, no numerical results are given in Sobolev's paper. It is easy to see, however, that for typical expansion velocities of two or three times the mean thermal velocity, the magnitude of the average internal radiation field will not be seriously affected, although the detailed frequency dependence will be modified. For example, if the expansion velocity increases linearly with optical depth from the inner boundary and becomesthree times the mean thermal velocity at the outer boundary, then roughly speaking, the optical thickness at any frequency in the line cannot be less than $\frac{1}{3}$ of its value in a static nebula, assuming Doppler broadening. Since the scattering is, in fact, noncoherent, the mean number of scatterings and therefore the average radiation 
field, is reduced by about the same factor. On the other hand, if the scattering were coherent, then this reduction would be by roughly a factor of 9 , which shows why velocity gradients appeared to be so important in the early studies. Capriotti (1965) has estimated that the mean probability of escape from a nebula with a line-centre optical thickness $10^{4}$ increases by only $50 \%$ as the expansion velocity at the outside increases from zero to three times the mean thermal velocity.

If one adopts an isothermal model of a planetary nebula and ignores the natural line width, the conclusion is that velocity gradients of the magnitude observed do not seriously alter the physical conditions within the nebula, although the details of the radiation field are affected.

Note added in proof. The accurate numerical solutions of the line transfer problem in cluding arbitrary velocity gradients recently obtained by Hummer and Rybicki (Astrophys. J., in press) verify the predictions of this section. Moreover, nebular shells expanding differentially towards the observer were found to cause red-shifted line profiles. This effect is probably that seen in Vaughan's observations of the $\lambda 10830$ line (these Proceedings and Astrophys. J., in press).

\subsubsection{THERMAL GRADIENTS}

According to presently accepted ideas, the electron and kinetic temperatures decrease toward the outer edge of the nebula, slowly in the $\mathrm{H}_{\mathrm{II}}$ region and very rapidly at the boundary between the $\mathrm{H}$ II and Hi regions. The general effect of such a temperature gradient is to reduce the opacity in the wings, thereby increasing the rate at which photons escape from the nebula. The effects of thermal gradients on resonance line transfer have been studied in detail by Hummer and Rybicki (1966) and Rybicki and Hummer (1967), who present extensive numerical results.

From an approximate solution of the nebular Ly- $\alpha$ problem with a thermal gradient, Pleškova (1962) found that such gradients as may plausibly exist in Hul regions have little influence on the $\mathrm{Ly}-\alpha$ radiation field in planetary nebulae. Even when an $\mathrm{H}$ l region is present, it can exert very little influence on the Ly- $\alpha$ radiation field, because the line opacity is concentrated strongly in the region of the line centre, while the radiation leaving the $\mathrm{HII}$ region is appreciable only in the line wings. Although Pleškova ignores the natural broadening wings, her conclusion is still essentially correct, for the following reason:

If we take the temperatures of the $\mathrm{HII}$ and $\mathrm{HI}$ regions to be $10^{4}{ }^{\circ} \mathrm{K}$ and $10^{2}{ }^{\circ} \mathrm{K}$, respectively, then the Doppler width in the $\mathrm{HI}_{\mathrm{I}}$ region is $1 / 10$ that in the $\mathrm{H}_{\mathrm{II}}$ region, and the ratios of natural to Doppler width are $a_{\mathrm{II}}=3.7 \times 10^{-4}$ and $a_{1}=3.7 \times 10^{-3}$. Since the optical thickness in Ly- $\alpha$ of the HII region is about $10^{4}$, most of the flux lies beyond three Doppler widths, measured in the Hil region, from the line centre and therefore beyond 30 Doppler widths, as measured in the Hi region. Using the asymp- 
totic form of the Voigt function, it is easy to show that the absorption coefficient for these frequencies is less than $2 \times 10^{-6}$ the line-centre value. Since there are no sources of $\mathrm{Ly}-\alpha$ in the Hi region, we can conclude that the Hi region has very little influence on the Ly- $\alpha$ field in planetary nebulae. This fact is also important in assessing the effects of velocity gradients, for it is sometimes argued that because scattering in the Hi region, occurring as it does predominantly in the far wings, is coherent, velocity gradients in the $\mathrm{HI}$ region have an important effect on the radiation field. However, since the line opacity in the relevant region is so small, this argument is irrelevant unless the extent of the HI region is enormous, i.e. $\tau_{0} \gg 10^{6}$. Even the shift of the linecentre opacity into the region of large flux by velocity gradients has little effect because the core in the Hi region is so narrow.

\subsubsection{INTERSTELLAR HYDROGEN}

If the interstellar neutral-hydrogen density is much lower than that in the outermost region of the nebula, the free path of an escaping photon will be much larger than it would be in the nebula, and simple geometrical arguments show that the photon has little chance of re-entering the nebula. On the other hand, if the nebula is completely ionized, then the neutral-hydrogen density may be of the same order of magnitude, so that a substantial effect could arise from the 'blanketing' by interstellar hydrogen.

\subsubsection{ABSORPTION MECHANISMS FOR Hi Ly- $\alpha$}

The radiation field in a line varies inversely as the sum of the mean probabilities per scattering of absorption and of escape. Thus, if the optical thickness of the nebula in the line is increased indefinitely, it is the most probable absorption process that finally limits the intensity of radiation in the line. As was first pointed out by Thomas (1949) and later by Burgess (1958), the assumptions of Baker and Menzel's (1938) Case $B$ are equivalent to setting both escape and absorption probabilities to zero, with the result that the populations of the $n=2$ levels are infinite. Provided that one is a priori sure that no upwards transitions occur from $n=2$, then no harm results from these assumptions in calculating the populations of the higher levels. On the other hand, if one wishes to check this point theoretically it becomes necessary to include the dominant escape and absorption processes in calculating the population of the $n=2$ levels.

It is also clear that the condition for 'detailed radiative balance' to occur, i.e. for upwards and downwards radiative transitions to balance sufficiently well for them to be regarded as exactly cancelling, is that the mean escape probability be smaller than the mean absorption probability. For the purposes of this discussion, we shall here take as standard conditions $T_{\mathrm{e}}=10^{4}{ }^{\circ} \mathrm{K}$ and $N_{e}=10^{4}{ }^{\circ} \mathrm{K}$. 


\subsubsection{MECHANISMS DESTROYING Ly- $\alpha$ IN SCATTERING}

Let us first examine possible mechanisms for depopulating the $2 p$ level before the emission of a further Ly- $\alpha$ photon occurs, i.e. for destroying a photon during the act of scattering. Spitzer and Greenstein (1951) first pointed out that collisional transitions from the $2 p$ to $2 s$ states, followed by two-quantum decay, could be important in this respect (cf. Seaton, 1955). The probability per scattering is

$$
p_{1}=\frac{N_{\mathrm{e}} q(2 \mathrm{p} \rightarrow 2 \mathrm{~s})}{A(2 \mathrm{p} \rightarrow 1 \mathrm{~s})} \cdot \frac{A_{2 q}(2 \mathrm{~s} \rightarrow 1 \mathrm{~s})+N_{\mathrm{e}} q(2 \mathrm{~s} \rightarrow 1 \mathrm{~s})}{A_{2 q}(2 \mathrm{~s} \rightarrow 1 \mathrm{~s})+N_{\mathrm{e}}(q(2 \mathrm{~s} \rightarrow 1 \mathrm{~s})+q(2 \mathrm{~s} \rightarrow 2 \mathrm{p}))},
$$

where we have assumed $N_{\mathrm{e}}=\mathrm{N}^{+}$. Here the $q$ 's are collisional rate constants and the $A$ 's are Einstein coefficients, with the subscript $2 q$ denoting two-quantum processes. Seaton (1955) gives $q(2 \mathrm{~s} \rightarrow 2 \mathrm{p})=3 q(2 \mathrm{p} \rightarrow 2 \mathrm{~s}) \approx 5 \times 10^{-4} \mathrm{~cm}^{3} \mathrm{sec}^{-1}$ for $1 \leqslant 10^{-4} T_{\mathrm{e}} \leqslant 2$. In the same temperature range, the expression given by Hummer (1963a) reduces to $q(2 \mathrm{p} \rightarrow 1 \mathrm{~s}) \sim 8 \times 10^{-9} \mathrm{~cm}^{3} \mathrm{sec}^{-1}$ and $q(1 \mathrm{~s} \rightarrow 2 \mathrm{p}) \sim 2.4 \times 10^{-8} \exp \left(-11.8 \times 10^{4} / T_{\mathrm{c}}\right)$ $\mathrm{cm}^{3} \mathrm{sec}^{-1}$. Using the values $A(2 \mathrm{p} \rightarrow 1 \mathrm{~s})=6.26 \times 10^{8} \mathrm{sec}^{-1}$ and $A_{2 q}(2 \mathrm{~s} \rightarrow 1 \mathrm{~s})=8.227 \mathrm{sec}^{-1}$, we find

$$
p_{1} \simeq \begin{gathered}
2.7 \times 10^{-13} N_{\mathrm{e}} \\
1+0.6 \times 10^{-4} N_{\mathrm{e}}
\end{gathered}, \quad 1 \leqslant 10^{-4} T_{\mathrm{e}} \leqslant 2 .
$$

At standard conditions, $p_{1} \simeq 2 \times 10^{-9}$. The failure of Yada $(1955 a, b)$ to obtain any reduction in the Ly- $\alpha$ flux by including this process in an approximate solution of the transfer problem for Lyman continuum optical thickness up to 10 is understandable, since the number of scatterings would have been at most $10^{6}$.

Collisional de-excitation of the $2 p$ level is also possible. The probability per scattering for this process is

$$
p_{2}=\frac{N_{\mathrm{e}} q(2 \mathrm{p} \rightarrow 1 \mathrm{~s})}{A(2 \mathrm{~s} \rightarrow 2 \mathrm{p})} \simeq 1.3 \times 10^{-17} N_{\mathrm{c}}, \quad 1 \leqslant 10^{-4} T_{\mathrm{e}} \leqslant 2 .
$$

At standard conditions, $p_{2} \simeq 1 \cdot 3 \times 10^{-13}$, which is negligible compared to $p_{1}$.

Kipper and Tiit (1958) have shown that in addition to the allowed $2 p \rightarrow 1 \mathrm{~s}$ transition there is also a small probability of two-quantum decay. They find, for the probability per scattering of a photon being destroyed in this way, $p_{3} \simeq 3 \times 10^{-14}$. Gurzadian (1961) has shown that $L y-\alpha$ can be scattered in magnetic dipole $1 \mathrm{~s} \rightarrow 2 \mathrm{~s}$ transitions, since the energy difference between the $2 s$ and $2 p$ states is less than the energy corresponding to a Doppler width. For the photon to be destroyed, two-photon decay must then occur. The probability per scattering for this mechanism is $p_{4} \simeq 5 \times 10^{-15}$. Gurzadian's original proposal involved a much larger decay rate for $2 \mathrm{~s}$, arising from a hypothetical electric dipole moment of the electron which was being discussed at the time. Subsequent experimental work has eliminated this possibility.

Collisional and radiative transitions from the $2 p$ to higher states are possible, although an atom thus excited will emit a photon that will, with high probability be 
degraded into another Ly- $\alpha$ photon, unless the nebula is very thin. The probability per scattering of collisional transitions from $2 \mathrm{p}$ to $3 \mathrm{~s}$ and $3 \mathrm{~d}$ is about $10^{-12}$, and that for radiative excitation by black-body radiation at $2.5 \times 10^{5}{ }^{\circ} \mathrm{K}$ and a dilution factor of $10^{-14}$ is about a factor of 10 smaller. Another possibility is the absorption of $\mathrm{HI} \mathrm{Ly}-\alpha$ in $2 \rightarrow 4$ transitions in $\mathrm{He}^{+}$. However, as the frequencies of the two lines differ by about 10 hydrogen Doppler widths, the probability of this process is also extremely small.

Kahn (1962) has suggested that since an $\mathrm{HI}$ Ly- $\alpha$ photon loses $10^{-8}$ of its energy, on the average, per scattering to atomic recoil, a photon could eventually diffuse out the red wing of the line. Under typical conditions, this would occur in about $10^{4}$ scatterings. However, because of the Doppler redistribution, as soon as the red side of the line becomes more intense, the number of red-to-blue scatterings increases to balance out the net energy loss to zero.

\subsubsection{MEChANISMS DESTROYING Ly- $\alpha$ BETWEEN SCATTERINGS}

Ly- $\alpha$ photons will be destroyed between scatterings by photo-ionizing atoms in states having an ionization potential less than $10 \cdot 15 \mathrm{eV}$. The highly populated metastable states of hydrogen and helium are the most likely to be important in this respect.

If $N_{\mathrm{A}}$ and $N_{\mathrm{S}}$ are the densities of absorbing and scattering atoms respectively, and $R_{\mathrm{A}}(v)$ and $R_{\mathrm{S}}(v)$ are the rates per unit frequency of absorbing and scattering radiation of frequency $v$, we can write

and

$$
R_{\mathrm{A}}(v) \mathrm{d} v=4 \pi N_{\mathrm{A}} a_{v}{ }_{h v}^{J} \mathrm{~d} v,
$$

$$
R_{\mathrm{S}}(v) \mathrm{d} v=N_{\mathrm{S}} B_{12} \phi(v) J_{v} \mathrm{~d} v
$$

where $a_{v}$ is the photo-ionization cross-section; $\phi(v)$ is the line-scattering coefficient normalized to unity; $\mathrm{B}_{12}$ is the Einstein coefficient for the scattering process; and $J_{v}$ is the mean intensity. For simplicity, assume that $J_{v}$ is constant over a frequency interval $2 \delta$ and zero elsewhere. Then the total rates are

and

$$
R_{\mathrm{A}}=\int_{v_{0}-\delta}^{v_{0}+\delta} R_{\mathrm{A}}(v) \mathrm{d} v \simeq 4 \pi N_{\mathrm{A}}^{v_{v_{0}}} 2 \delta v_{0} J_{v_{0}},
$$

$$
R_{\mathrm{S}}=\int_{v_{0}-\delta}^{v_{0}-\delta} R_{\mathrm{S}}(v) \mathrm{d} v \simeq N_{\mathrm{S}} B_{12} J_{v_{0}},
$$

and the probability of absorption per scattering is

$$
p_{\mathrm{A}}=\begin{array}{ll}
R_{\mathrm{A}} \\
R_{\mathrm{S}}
\end{array}=\begin{array}{ll}
8 \pi \delta a_{v_{0}} & N_{\mathrm{A}} \\
h v_{0} B_{12} & N_{\mathrm{S}}
\end{array} .
$$


The half-width $\delta$ is typically 3 Doppler half-widths. Using the numerical values for HI Ly- $\alpha, \Delta_{0}=1.06 \times 10^{11} \mathrm{sec}^{-1} ; h v_{0} B_{12}=0.14 \mathrm{~cm}^{2} \mathrm{sec}$, we have

$$
p_{\mathrm{A}}=5 \times 10^{13} \frac{N_{\mathrm{A}}}{N_{\mathrm{S}}} a_{v 0} .
$$

The photo-ionization cross-sections at $\mathrm{Ly}-\alpha$ of $\mathrm{H}(2 \mathrm{~s})$ and $\mathrm{He}\left(2^{3} \mathrm{~S}\right)$ are approximately $6 \times 10^{-19} \mathrm{~cm}^{2}$ (Seaton, 1960) and $1.5 \times 10^{-18} \mathrm{~cm}^{2}$ (Huang 1948), respectively. We can estimate the $\mathrm{H}(2 \mathrm{~s})$ population by balancing recombination against two-quantum decay and $2 \mathrm{~s} \rightarrow 2 \mathrm{p}$ transitions, assuming $N_{\mathrm{e}}=N_{+}$,

$$
\frac{1}{3} \alpha_{B} N_{\mathrm{e}}^{2}=N(2 \mathrm{~s})\left[A_{2 q}(2 \mathrm{~s} \rightarrow 1 \mathrm{~s})+N_{\mathrm{e}} q(2 \mathrm{~s} \rightarrow 2 \mathrm{p})\right],
$$

or using the numerical values given above,

$$
N(2 \mathrm{~s})=\frac{1 \times 10^{-14} N_{\mathrm{e}}^{2}}{1+0.6 \times 10^{-4} N_{\mathrm{e}}} .
$$

As the population of $\mathrm{He}\left(2^{3} \mathrm{~S}\right)$ is affected by a number of processes, we shall infer its value from O'Dell's (1965) measures of the ratio $I(10830) / I(5876)$. Since the $2^{3} \mathrm{P}$ level is populated by collisional excitations from $2^{3} \mathrm{~S}$ and by recombination, and depopulated mainly by radiative transitions to $2^{3} \mathrm{~S}$, we have (cf. O'Dell, 1965)

$$
I(10830) / I(5876)=\frac{5876}{10830} \frac{N\left(2^{3} \mathrm{~S}\right) q\left(2^{3} \mathrm{~S} \rightarrow 2^{3} \mathrm{P}\right)+\frac{1}{2} \alpha_{B} N\left(\mathrm{He}^{+}\right)}{N\left(\mathrm{He}^{+}\right) \alpha_{\mathrm{eff}}(5876)} .
$$

Using the values given by Seaton (1968) for $T_{\mathrm{e}}=10^{4}{ }^{\circ} \mathrm{K}, q\left(2^{3} \mathrm{~S} \rightarrow 2^{3} \mathrm{P}\right) \sim 2 \times 10^{-7}$ $\mathrm{cm}^{3} \mathrm{sec}^{-1}$, and $\alpha_{\mathrm{eff}}(5876)=5.2 \times 10^{-14}$ and taking $\frac{1}{2} \alpha_{B}=1 \cdot 3 \times 10^{-14}$, we have

$$
\frac{N\left(2^{3} \mathrm{~S}\right)}{N\left(\mathrm{He}^{+}\right)}=2.6 \times 10^{-7}\left[1.8 \frac{I(10830)}{I(5876)}-2.5\right] \text {. }
$$

O'Dell's values of $I(10830) / I(5876)$ lie between $1 \cdot 9$ and $9 \cdot 6$, so that $N\left(2^{3} \mathrm{~S}\right) / N\left(\mathrm{He}^{+}\right)$ lie between $4 \times 10^{-6}$ and $2 \times 10^{-7}$. In the region where the helium lines are formed $N\left(\mathrm{He}^{+}\right) \sim N_{\mathrm{He}} \sim \frac{1}{5} N_{\mathrm{H}} \sim 2 \times 10^{3} \mathrm{~cm}^{-3}$. Thus for the probabilities per scattering of absorption of $\mathrm{HI}_{\mathrm{I}} \mathrm{Ly}-\alpha$ by $\mathrm{H}(2 \mathrm{~s})$ and $\mathrm{He}\left(2^{3} \mathrm{~S}\right)$, we have

and

$$
p_{\mathrm{A}}(\mathrm{H} 2 \mathrm{~s})=2 \times 10^{-11} / N_{\mathrm{s}}
$$

$$
3 \times 10^{-8} \leqslant N_{\mathrm{s}} p_{\mathrm{A}}\left(\mathrm{He} 2^{3} \mathrm{~S}\right) \leqslant 6 \times 10^{-7} .
$$

Since $N_{S}=N(\mathrm{H} \mathrm{1s})$ is hardly smaller than $1 \mathrm{~cm}^{-3}$, absorption by $\mathrm{H}(2 \mathrm{~s})$ is unimportant, while absorption by $\mathrm{He}\left(2^{3} \mathrm{~S}\right)$ appears to be the dominant loss mechanism, at least in regions where helium is mostly singly ionized and $N(\mathrm{H} 1 \mathrm{~s}) \lesssim 10^{2} \mathrm{~cm}^{-3}$.

Finally, there is the possibility of extinction by dust grains. Although there is at present no direct observational evidence for dust in planetary nebulae, quantities of dust otherwise unobservable could still play a role in destroying Ly- $\alpha$ because of the very 
long paths of these photons in the nebula. If planetary nebulae do, in fact, evolve from red giants, then it is not surprising that dust should exist in at least the $\mathrm{HI}_{\mathrm{I}}$ regions of planetaries. Any energy absorbed by dust would be re-emitted in the infrared; whether or not the strong infrared continuum observed in NGC 7027 by Gillett et al. (1967) has its origin in this mechanism remains a matter of speculation.*

\subsubsection{Absorption mechanisms for Hei AND Heil Ly- $\alpha$}

The Ly- $\alpha$ lines of both HeI and HeII are capable of ionizing hydrogen, and that of HeII can ionize helium. Using the formula for $p_{\mathrm{A}}$ derived above, and the numerical values for He II Ly- $\alpha ; \Delta=2 \cdot 1 \times 10^{11} \mathrm{sec}^{-1}, h v_{0} B_{12}=0 \cdot 14 \mathrm{~cm}^{2} \mathrm{sec}^{-1} ;$ and $a_{v_{0}}=2 \cdot 9 \times 10^{-19}$ $\mathrm{cm}^{2}$, we obtain for the probability per scattering that a He II Ly- $\alpha$ photon is absorbed by hydrogen,

$$
p_{\mathrm{A}}(\mathrm{H}) \sim 3.3 \times 10^{-5} \frac{N(\mathrm{H} 1 \mathrm{~s})}{N\left(\mathrm{He}^{+} 1 \mathrm{~s}\right)} .
$$

From Goodson's (1967) ionization equilibria we find values of $N(\mathrm{H} 1 \mathrm{~s}) / N\left(\mathrm{He}^{+} 1 \mathrm{~s}\right)$ on the order of $10^{-4}-10^{-2}$ throughout the region of interest, so that $p_{\mathrm{A}}(\mathrm{H}) \sim 10^{-9}-10^{-7}$. The probability per scattering of absorption of $\mathrm{He}$ I Ly- $\alpha$ by hydrogen is similar. The probability that a HeII Ly- $\alpha$ photon is absorbed in a $2 \mathrm{p}^{2}{ }^{3} \mathrm{P}_{2} \rightarrow 2 \mathrm{p} 3 \mathrm{~d}{ }^{3} \mathrm{P}_{2}^{0}$ (the initial transition in the Bowen mechanism) is difficult to estimate because it will depend on the details of the radiation field which can be obtained only by a solution of the transfer equation, but it could easily be important because of the resonant nature of the absorption. On the other hand, the probability per scattering of loss by $2 \mathrm{p} \rightarrow 2 \mathrm{~s}$ transitions in $\mathrm{He}^{+}$is about a factor of 70 smaller than for $\mathrm{H}$ (Hummer, 1963b).

\subsubsection{Possible Consequences of large Ly- $\alpha$ intensities}

Historically, the first important consequence of Ly- $\alpha$ intensity to be considered was the catastrophic radiation pressure. The calculations of Koelbloed (1956) and of Zanstra (1956) show that for a total thickness of $2 \times 10^{4}$, the inclusion of noncoherent scattering reduces the radiation pressure by a factor between 40 and 500, depending on position, from the value with coherent scattering, with the result that Ly- $\alpha$ radiation pressure is not of dynamical importance. Moreover, as we have argued above, any increase in the optical thickness in Ly- $\alpha$ beyond $10^{4}$ must come from an $\mathrm{HI}$ region, in which the hydrogen line is so narrow as to miss most of the radiation escaping from the $\mathrm{H}_{\mathrm{II}}$ region. It would appear that $\mathrm{HI}_{\mathrm{I}} \mathrm{Ly}-\alpha$ radiation pressure is unimportant until the Ly- $\alpha$ optical thickness exceeds $10^{7}$.

If the $L y-\alpha$ radiation becomes very intense, the population of the $2 p$ level may become sufficiently large so that absorption of the Balmer lines and collisional transi-

* Krishna Swamy and O'Dell (Astrophys. J., 151, L61, 1968) have presented impressive evidence in support of this mechanism. 
tions from this level become important. $\mathrm{H}$ I Ly- $\alpha$ could also play a role in depopulating the $2^{3} \mathrm{~S}$ state in HeI.

\subsubsection{ABSORPTION IN THE BaLMER LINES}

In his original investigations of the Ly- $\alpha$ radiation field, Ambartsumian $(1932,1933)$ found that the $2 \mathrm{p}$ population was large enough for the Balmer $\alpha$ and $\beta$ lines to be optically thick. Pottasch $(1960 a, b)$, who included in his calculations the effects of noncoherent scattering, obtained smaller excited state populations than Ambartsumian, but concluded that self-absorption could occur in the early Balmer lines. However, because his frequency-quadrature scheme had artificially impaired the escape of Ly- $\alpha$ radiation, Pottasch obtained values of the $2 p$ population considerably too large. Osterbrock's (1962) calculations showed that for typical nebulae self-absorption was probably negligible. Gershberg (1961) and Mathis (1962) reached the same conclusion from observational evidence.

Capriotti $(1964 a, b)$ has calculated the Balmer decrement as a function of the optical thickness in the $2 \mathrm{p} \rightarrow 3 \mathrm{~s}, 3 \mathrm{~d}$ transitions and the $2 \mathrm{~s} \rightarrow 3 \mathrm{p}$ transition. An examination of the observed Balmer $\alpha, \beta, \gamma$ ratios in the light of Capriotti's results indicates that selfabsorption in the Balmer lines is completely unimportant for the overwhelming majority of nebulae for which we have observations.

The effect of self-absorption on the triplet system of helium has been discussed most recently by Pottasch (1962) and by Osterbrock (1964), who give references to earlier work. It appears that self-absorption is important in some objects, although other mechanisms may also be operative.

\subsubsection{COLLISIONAL PROCESSES FROM $n=2$}

Jefferies and Pottasch (1959) and Pottasch (1960c) found that for optically thick nebulae the populations of the excited states of hydrogen are so large that collisional ionization from these states are comparable in number with ground state photo-ionizations. Consequently the generation of diffuse photons is increased substantially by the inclusion of these processes, and the ionized region is considerably larger than the Strömgren estimate. However, if this effect exists, it must arise primarily from the $n=2$ level, since the populations of the higher states are much smaller, while their collisional ionization rate constants are not much larger than those for $n=2$. An upper limit to the $2 p$ population is obtained by setting the escape probability to zero and assuming that $2 \mathrm{p} \rightarrow 2 \mathrm{~s}$ transitions followed by two-quantum decay are the dominant absorption mechanism, with a probability per scattering of $10^{-9}$ (see Section 3.2.0). Then the maximum density of atoms in the $2 \mathrm{p}$ state is given approximately by (Osterbrock, 1964)

$$
10^{-9} A(2 \mathrm{p} \rightarrow 1 \mathrm{~s}) N_{\max }(2 \mathrm{p})=N_{\mathrm{e}} N_{+} \frac{2}{3} \alpha_{B}
$$


or assuming $N_{\mathrm{e}}=N_{+}=10^{4}$,

$$
N_{\max }(2 \mathrm{p}) \sim 2 \times 10^{-5} \mathrm{~cm}^{-3} .
$$

The $2 \mathrm{~s}$ population is about $10^{-6}$ (Section 3.2.2) in these conditions. Rudge and Schwartz (1966) give the ionization rate constant for $2 \mathrm{~s}$ as $6.8 \times 10^{-9} \mathrm{~cm}^{3} \mathrm{sec}^{-1}$ at $T_{\mathrm{e}}=10^{4}$, and since Prasad (1966) has shown that the $2 \mathrm{~s}$ and $2 \mathrm{p}$ ionization cross-sections are not very different at low energies, we see that the maximum collisional ionization rate from $n=2$ is about $10^{-9} \mathrm{sec}^{-1}$, while the photo-ionization rate from $1 \mathrm{~s}$ is about $10^{-5} \mathrm{sec}^{-1}$. It would appear that collisional ionization from excited states is completely unimportant for planetary nebulae.

Pottasch (1960a) has also investigated the possible role of collisional excitation from the $n=2$ states and finds that it is negligible by several orders of magnitude, even using his overestimated $n=2$ populations.

\subsubsection{Photo-ionization of He $2^{3} \mathrm{~S}$ вy Hi Ly- $\alpha$}

The photo-ionization of $\mathrm{He}\left(2^{3} \mathrm{~S}\right)$ by $\mathrm{HI}$ Ly- $\alpha$ has already been discussed (Section 3.2.2) as a destruction mechanism for Ly- $\alpha$. Münch has suggested that these processes could be important in controlling the population of $\mathrm{He}\left(2^{3} \mathrm{~S}\right)$. O'Dell (1965) has investigated this problem on the assumption that $2^{3} \mathrm{~S}$ is populated by recombination (including cascade) and is depopulated by two-photon transitions to $1^{1} \mathrm{~S}$, photoionization by stellar radiation, collisional transitions to $2^{1} \mathrm{~S}$, and photo-ionization by Ly- $\alpha$. From the observed intensity ratios of the lines $\mathrm{He} \mathrm{I} \lambda \lambda 10830 \AA$ and $3889 \AA$ to $\lambda 5876 \AA$, O'Dell derives a measure of the distance traveled by a typical Ly- $\alpha$ photon in the nebula, much larger than would be estimated from Osterbrock's mean number of scatterings for the Hir zone. O'Dell suggests that this is evidence for an extensive neutral-hydrogen region blanketing the Hil region. However, O'Dell's $2^{3} \mathrm{~S} \rightarrow 2^{1} \mathrm{~S}$ collision rate constant is too small by a factor of 5 , and his value for the $2^{3} \mathrm{~S} \rightarrow 2^{3} \mathrm{P}$ is too large by a factor of $1 \cdot 7$ (Seaton, 1968). He also neglected collisional transitions to $2^{1} \mathrm{P}$ and what is perhaps the most important mechanism, ${ }^{*}$ photo-ionization by the entire Balmer spectrum of HeII. All of these factors tend to make O'Dell's estimate of the ionizing power of $\mathrm{HI} \mathrm{Ly}-\alpha$ too large.

\subsubsection{EfFects of Heil Ly- $\alpha$}

Probably the most important role played by this radiation is in ionizing hydrogen and helium. It is clear from Goodson's (1967) work that HeII Ly- $\alpha$ makes a major contribution to the energy balance, so that a careful solution of this transfer problem

* Robbins (Astrophys. J., 151, L35, 1968) has suggested that excitation of doublyexcited autoionizing states in helium by ultraviolet lines of CIII and NIV may be important in depopulating the $2^{3} \mathrm{~S}$ level of Her. 
along with the ionization balance of $\mathrm{H}$ and $\mathrm{He}$ is important in determining the correct run of electron temperature with depth. In particular, it is important to know whether $\mathrm{He}$ II $\mathrm{Ly}-\alpha$ is absorbed in the $\mathrm{He}^{+2}$ region, or whether it escapes and is absorbed in regions where the densities of $\mathrm{H}$ and $\mathrm{He}$ are larger. Since the electrons produced by the photo-ionization of $\mathrm{H}$ and $\mathrm{He}$ have energies of 27.2 and $16.2 \mathrm{eV}$ respectively, which are much larger than the mean electron energy $(\sim 1 \mathrm{eV})$, their thermalization will occur much more slowly than would be expected from the estimates of Böhm and Aller (1947). If these electrons suffered inelastic collisions with atoms before being thermalized, a substantial part of their energy could escape as radiation and should not be included in the thermal balance.

\subsection{Transfer Effects and the Hydrogen Recombination Spectrum}

The Lyman lines beyond Ly- $\alpha$ have an entirely different character from Ly- $\alpha$ because their photons have a large probability per scattering $(\gtrsim 0.5)$ of being degraded into photons in a lower Lyman line and a subordinate line. For this reason the population of the excited levels other than $n=2$ varies little with optical thickness.

Since the optical thicknesses of the Lyman lines have fixed ratios, the populations of the excited states should increase monotonically from their Case A to Case B values as the optical thickness is increased, assuming self-absorption in the Balmer line is negligible. Since, however, the level populations will increase at different rates, the ratios of Balmer line intensities need not necessarily lie between their Case A and Case B values. Capriotti (1966) has investigated the effect on the relative intensities of Balmer $\alpha, \beta, \gamma$ and $\delta$ of allowing for the partial escape of Lyman photons. The escape probabilities was estimated by an extension of Zanstra's argument (Section 3.1.1) to a nebula expanding with a constant velocity gradient. The line-intensity ratios, displaced by an arbitrary amount along the reddening curve, show better agreement with the observed ratios than do either the Case A or Case B values, although by no means all observed ratios lie within Capriotti's bounds. It is also not clear that arguments based on averages of quantities that vary by orders of magnitude are an entirely reliable basis on which to discuss variations in line ratios on the order of 0.03 in the logarithm. Since upward transitions from excited states are negligible, each Lyman line transfer problem* can be solved successively, starting with a high, optically thin line, and working towards Ly- $\alpha$. This is quite feasible with modern computing technique and a definite answer to this problem should be available.

* Van Blerkom and Hummer (Astrophys. J., in press) have shown the importance of overlapping on the transfer problem of the higher Lyman lines and have developed a simple band model for the inclusion of these effects. 


\section{Acknowledgements}

I am grateful to Professor M.J. Seaton, Dr John Stewart, Dr G. Khromov, and Mr D. Van Blerkom for many discussions which have helped to clarify my understanding of various aspects of the material discussed here, and to Dr L. H. Auer for giving me his Monte-Carlo results prior to publication.

\section{References}

Aller, L.H., Baker, J.G., Menzel, D.H. (1939) Astrophys. J., 90, 601.

Ambartsumian, V.A. (1932) Mon. Not. R. astr. Soc., 93, 50.

Ambartsumian, V.A. (1933) Bull. Obs. centr. Poulkovo, 13, 3.

Auer, L.H. (1968) Astrophys. J., 153 (in press).

Baker, J.G., Menzel, D.H. (1938) Astrophys. J., 88, 52.

Baker, J.G., Aller, L.H., Menzel, D.H. (1939) Astrophys. J., 90, 271.

Biberman, L. M. (1948) Dokl. Akad. Nauk. SSSR, 49, 659.

Biberman, L. M., Vorobyev, V.S., Lagarkov, A.N. (1965) Optika Spektrosk., 19, 326; trans. Optics Spectrosc., 19, 186.

Böhm, D., Aller, L.H. (1947) Astrophys. J., 105, 1.

Böhm, K.-H., Deinzer, W. (1965) Z. Astrophys., 61, 19.

Böhm, K.-H., Deinzer, W. (1966) Z. Astrophys., 63, 177.

Burgess, A. (1958) Mon. Not. R. astr. Soc., 118, 477.

Capriotti, E.R. (1964a) Astrophys. J., 139, 225.

Capriotti, E.R. (1964b) Astrophys. J., 140, 632.

Capriotti, E.R. (1965) Astrophys. J., 142, 1101.

Capriotti, E.R. (1966) Astrophys. J., 146, 709; cf. erratum, ibid., 148, 1967, 318.

Chamberlain, J.W. (1953) Astrophys. J., 117, 387.

Chandrasekhar, S. (1935) Z. Astrophys., 9, 266.

Eberlein, K. (1955) Z. Astrophys., 38, 14.

Eberlein, K. (1957) Z. Astrophys., 41, 271.

Field, G.B. (1959) Astrophys. J., 129, 551.

Gershberg, R. (1961) Astr. Zu., 38, 250; trans. Soviet Astr., 5, 188.

Gillett, F.C., Low, F. J., Stein, W.A. (1967) Astrophys. J., 149, L97.

Goodson, W.L. (1967) Z. Astrophys., 66, 118.

Gurzadian, G.A. (1961) Dokl. Akad. Nauk. SSSR., 141, 1061; trans. Soviet Phys. Dokl., 6, 1031, 1962.

Harman, R.J., Seaton, M.J. (1966) Mon. Not. R. astr. Soc., 132, 15.

Hearn, A. G. (1964) Proc. Phys. Soc., London, 84, 11.

Henyey, L.G. (1941) Proc. nat. Acad. Sci. Am., 26, 50.

Hjellming, R. (1966) Astrophys. J., 143, 420.

Huang, S.S. (1948) Astrophys. J., 108, 354.

Hummer, D. G. (1962) Mon. Not. R. astr. Soc., 125, 21.

Hummer, D. G. (1963a) Mon. Not. R. astr. Soc., 125, 461.

Hummer, D.G. (1963b) Thesis, Univ. of London.

Hummer, D.G. (1964) Astrophys. J., 140, 276.

Hummer, D. G. (1968) Mon. Not. R. astr. Soc., $138,73$.

Hummer, D.G., Rybicki, G.B. (1966) J. quantit. Spectrosc. radiat. Transfer, 6, 661.

Hummer, D.G., Rybicki, G.B. (1967) Computational Methods for Non-LTE Line-Transfer Problems, in Methods in Computational Physics, 7, Ed. by B. Alder, S. Fernbach, M. Rotenberg, Academic Press, New York, p. 53.

Hummer, D. G., Seaton, M.J. (1963) Mon. Not. R. astr. Soc., $125,437$.

Hummer, D. G., Seaton, M.J. (1964) Mon. Not. R. astr. Soc., 127, 217. 
Ivanov, V.V. (1967) Bull. astr. Inst. Netherl., 19, 192.

Jefferies, J. T., Pottasch, S. R. (1959) Ann. Astrophys., 22, 318.

De Jong, J.H. (1951) Bull. astr. Inst. Netherl., 11, 345.

Kahn, F. (1962) Dynamics of Interstellar Gas, in Interstellar Matter in Galaxies, Ed. by L. Woltjer, W. A. Benjamin, Inc., New York, p. 164.

Kipper, A. Ya., Tiit, V. M. (1958) Vop. Kosmog., 6, 99.

Koelbloed, D. (1956) Bull. astr. Inst. Netherl., 12, 341.

Mathis, J. (1962) Astrophys. J., 136, 374.

O’Dell, C.R. (1965) Astrophys. J., 142, 1093.

Osaki, T. (1962) Publ. astr. Soc. Japan, 14, 111.

Osterbrock, D.E. (1962) Astrophys. J., 135, 195.

Osterbrock, D.E. (1964) A. Rev. Astr. Astrophys., $2,95$.

Parker, R.A.R. (1964) Astrophys. J., 139, 208.

Pleškova, T.F. (1962) Astr. Zu., 39, 235; trans. Soviet Astr., 6, 182.

Pottasch, S.R. (1960a) Astrophys. J., 131, 202.

Pottasch, S.R. (1960b) Ann. Astrophys., 23, 749.

Pottasch, S.R. (1960c) Astrophys. J., 132, 269.

Pottasch, S.R. (1962) Astrophys. J., 135, 385.

Prasad, S.S. (1966) Proc. phys. Soc., London, 87, 393.

Rudge, M.R.H., Schwartz, S. B. (1966) Proc. phys. Soc., 88, 563.

Rybicki, G. B., Hummer, D.G. (1967) Astrophys. J., 150 , 607.

Seaton, M.J. (1955) Mon. Not. R. astr. Soc., 115, 279.

Seaton, M.J. (1960) Rep. Prog. Phys., 23, 313.

Seaton, M.J. (1968) Review of Atomic Collision Processes, in the present volume, p. 129.

Sobolev, V.V. (1944) Astr. Zu., 21, 143.

Sobolev, V.V. (1947) Dvižuščiesja Oboločki Zvezd, Leningrad; trans. Moving Envelopes of Stars, translated from the Russian by S. Gaposchkin, Harvard University Press, Cambridge, Mass., 1960. Sobolev, V.V. (1957) Astr. Zu., 34, 694; trans. Soviet Astr., 1, 678.

Sobolev, V.V. (1967) Astrofizika, 3, 137.

Spitzer, L., Greenstein, J.L. (1951) Astrophys. J., 114, 407.

Strömgren, B. (1939) Astrophys. J., 89, 526.

Thomas, R.N. (1949) Astrophys. J., 109, 480.

Unno, W. (1951) Publ. astr. Soc. Japan, 3, 158.

Unno, W. (1955) Publ. astr. Soc. Japan, 7, 81.

Van Blerkom, D. (1968) Astrophys. J., 152 (in press).

Van Blerkom, D., Hummer, D.G. (1967) Mon. Not. R. astr. Soc., 137, 353.

Yada, B. (1955a) Publ. astr. Soc. Japan, 5, 128.

Yada, B. (1955b) Publ. astr. Soc. Japan, 6, 76.

Zanstra, H. (1934) Mon. Not. R. astr. Soc., 95, 84.

Zanstra, H. (1936) Mon. Not. R. astr. Soc., 97, 37.

Zanstra, H. (1949) Bull. astr. Inst. Netherl., 11, 1.

Zanstra, H. (1951a) Bull. astr. Inst. Netherl., 11, 341.

Zanstra, H. (1951b) Bull. astr. Inst. Netherl., 11, 359.

Zanstra, H. (1956) Bull. astr. Inst. Netherl., 12, 349.

\section{DISCUSSION}

Böhm: I agree with Hummer's statement that in principal the treatment of the He II resonance radiation in Goodson's paper is not satisfactory. On the other hand the numerical values calculated by Goodson for the electron temperature in the core of the nebula lie between $2 \times 10^{4}$ and $2.2 \times 10^{4}$ ${ }^{\circ} \mathbf{K}$, and essentially agree with the numbers given by Flower.

Seaton: The agreement between Goodson and Flower is a consequence of their having made the same assumptions. 